\title{
ОБ ОДНОМ МЕТОДЕ СИНТЕЗА ОБОБЩЕННОГО КРИТЕРИЯ ЭФФЕКТИВНОСТИ В СИСТЕМАХ АВТОМАТИЗИРОВАННОГО ПРОЕКТИРОВАНИЯ АГРЕГАТНОГО ОБОРУДОВАНИЯ
}

\author{
(Представил Б. Тамм)
}

\section{1. Исходные предпосылки}

В большинстве существующих работ по автоматизированному проектированию оценка результатов проектирования производится по одному скалярному, легко формализуемому критерию $\left[{ }^{1-4}\right]$, либо по многим критериям с использованием ступенчатого процесса принятия решений $\left[{ }^{5,6}\right]$. Такой подход относительно прост в реализации, однако применение его в системах автоматизированного проектирования агрегатного оборудования, которым свойственны сложные модели и большое число разнообразных (нередко даже противоречивых) критериев, не приводит к оптимальным решениям.

Одним из подходов, позволяющим принимать решения по нескольким, в том числе и противоречивым критериям, является оценивание по векторным критериям эффективности проектирования [ $\left.{ }^{7}\right]$. Критерии эффективности используются при построении оценок качества проектных решений как в целом по задаче, так по отдельным подзадачам проектирования. Большинство известных в настоящее время подходов к решению задач проектирования с векторными критериями предусматривают использование информации о важности критериев для синтеза на ее основе обабщенного критерия $\left[{ }^{8-10}\right]$.

Обобщенный критерий эффективности, будучи скалярной функцией частных критериев, учитывает степень достижения всех целей в совокупности, отражая их относительную значимость исходя из общей цели проектирования агрегатного оборудования. Часто в качестве такой общей цели выбирается один из критериев проектирования, например критерий минимальной стоимости $\left[{ }^{11,12}\right]$. При этом коэффициенты важности частных критериев проектирования задаются исходя из степени их влияния на оценку по обобщенному критерию.

Задание коэффициентов важности в обобщенном критерии проектирования достаточно сложный и трудоемкий процесс, причем информация об этих коэффициентах задается не всегда достаточно обоснованно $\left[{ }^{13}\right]$. Трудности определения коэффициентов важности задачах автоматизированного проектирования привели к широкому распространению проектирования по прототипу $\left[{ }^{14}\right]$. При этом уменьшается вероятность получения неудачного решения, однако отсутствие новизны не позволяет разрабатывать более эффективное оборудование по сравнению с выпущенным ранее. 
Другим подходом в оптимальном проектировании оборудования является статистическое моделирование оценочной функции обобщенного критерия с использованием регрессионного и корреляционного анализов [ $\left.{ }^{11}\right]$. Этот подход более перспективен для систем автоматизированного проектирования агрегатного оборудования, так как он сочетает в себе статистический анализ множества ранее разработанных проектов (а именно в таких случаях достигается высокая степень унификации и агрегатирования) и возможность принимать более правильное решение в условиях изменяющейся от проекта к проекту общей цели проектирования.

В данной работе рассматриваются методы построения весовых оценок обобщенного критерия проектирования в условиях изменяющейся общей цели проектирования, проводятся статистический анализ и прогнозирование значений обобщенного критерия, строится обобщенная оценочная функция, учитывающая сложность проектируемого оборудования.

\section{2. Постановка задачи построения обобщенного критерия}

Большинство методов многокритериальной оптимизации предполагают построение обобщенного критерия путем «свертывания» множества критериев $K=\left\{K_{1}, K_{2}, \ldots, K_{p}\right\}$ в одну функцию $\dot{K}=\Phi\left(\alpha_{1}, K_{1}, \ldots\right.$ $\left.\ldots, \alpha_{p} K_{p}\right\}$, где $\alpha_{i}-$ коэффициенты относительной важности частных критериев $\left[{ }^{7,9}, 10,15\right]$. В результате задача многокритериальной оптимизации сводится к задаче оптимизации по одному главному критерию проектирования агрегатного оборудования, остальные же являются частными критериями и оценка по ним функционально подчиняется оценке по главному. Примем в качестве такового один из критериев, оценка по которому может быть получена только после проектирования (напр., минимум веса, стоимости, эксплуатационных затрат и т. д.). Такой подход в задании главного критерия проектирования широко используется в традиционном ручном проектировании, он естествен и удобен для конструктора $\left[{ }^{16,17}\right]$.

Оценки значений главного критерия проектирования могут быть получены как до процесса проектирования - априорные, или прогнозируемые, оценки, так и после него - апостериорные, или конструктивные, оценки качества проектирования.

Априорное оценивание значений главного критерия проектирования состоит в анализе исходной, по отношению к процессу проектирования, информации, получаемой из технического задания на проектирование агрегатного оборудования, и в построении на основе этой информации прогноза значений главного критерия.

Представим прогноз оценки главного критерия $X^{\text {п }}$ выражением вида

$$
X^{\mathrm{I}}=\psi\left(\alpha_{i}, X_{i}\right), \quad i=\overline{1, n},
$$

где $X_{i}$ - оценки по частным критериям; $\psi-$ допустимое преобразование частных оценок.

Уравнение (1) оценивает главный критерий проектирования в зависимости от сложности технического задания на проектирование агрегатного оборудования.

По аналогии с уравнением (1) можно записать уравнение оценочной шкалы по главному критерию при апостериорных, конструктивных, оценках: 


$$
X^{\mathrm{\kappa}}=\varphi\left(\beta_{j}, X_{j}\right), \quad j=\overline{1,(p-1)}, \quad p-1>n, \quad i \neq j,
$$

где $X_{j}$ - оценки по частным критериям, $\beta_{j}-$ коэффициенты важности частных критериев относительно главного критерия, $\varphi$ - допустимое преобразование частных оценок.

Пусть цель оптимального проектирования агрегатного оборудования состоит в том, чтобы при заданной по $X^{\text {п }}$ сложности задания на проектирование получить исходную 'конструктивную оценку $X^{\kappa}$ такую, которая бы при минимизации главного критерия не превышала прогнозируемую, а при максимизации его была не меныше прогнозируемой. Тогда справедливы следующие выражения:

$$
\begin{aligned}
& \eta_{x}=X^{\mathrm{n}} / X^{\kappa}, \\
& \eta_{x} \geqslant 1, \text { если }\left(x_{j} \in X^{\kappa}\right) \rightarrow \max , \\
& \eta_{x} \leqslant 1, \text { если }\left(x_{j} \in X^{\kappa}\right) \rightarrow \min .
\end{aligned}
$$

В противном случае оцениваемый по обобщенному критерию вариант конструкции оборудования следует отвергнуть и продолжить поиск решения, удовлетворяющего условиям (4) или (5).

\section{3. Статистическое исследование агрегатного оборудования}

Приступая к статистическому исследованию ранее разработанного оборудования, следует выбирать такие его параметры, которые будут служить оценками по критериям проектирования или самими критериями. В качестве оценок берутся параметры агрегатного оборудования, характеризующие его в целом: производительность, масса, стоимость, размерные характеристики и т. д.

Представим множество критериев проектирования $K$ в виде двух подмножеств $K=\left\{K^{s}, K^{k}\right\}$, где $K^{s}-$ критерии, оценивающие исходные параметры оборудования, значения которых известны из технического задания на проектирование, $K^{k}$ - критерии, оценивающие конечные конструктивные параметры агрегатного оборудования $x_{m}, x_{m+1}, \ldots, x_{p}$ при $m=p-n$. По результатам проведенной классификации параметров строятся таблицы, куда заносятся данные о параметрах агрегатного оборудования (см. ниже).

Наблюдаемые проекты агрегатного оборудования должны быть статистически независимы и должны выбираться случайным образом, например по таблице случайных чисел. Количество принимаемых к рассмотрению проектов зависит от допустимой ошибки на полноту выборки. Так, при $180-200$ проектах ошибка $\varepsilon=0,05$ при величине вероятности $P=0,85\left[{ }^{18}\right]$. С помощью метода наименьших квадратов находятся средние значения и дисперсии оценочных параметров оборудования

$$
\begin{aligned}
& M\left[x_{i}\right]=k^{-1} \sum_{0=1}^{k} x_{i 0}, \quad M\left[x_{j}\right]=k^{-1} \sum_{0=1}^{k} x_{j 0} ; \\
& \sigma_{i}^{2}=(k-1)^{-1} \sum_{\theta=1}^{k}\left(x_{i \vartheta}-M\left[x_{i \vartheta}\right]\right)^{2}, \sigma_{j}^{2}=(k-1)^{-1} \sum_{\theta=1}^{h}\left(x_{j \vartheta}-M\left[x_{j \diamond}\right]\right)^{2} .
\end{aligned}
$$

Коэффициенты парной корреляции для различных видов связи оценочных параметров вычисляются по следующим формулам: 
Таблица параметров ранее разработанного агрегатного оборудования

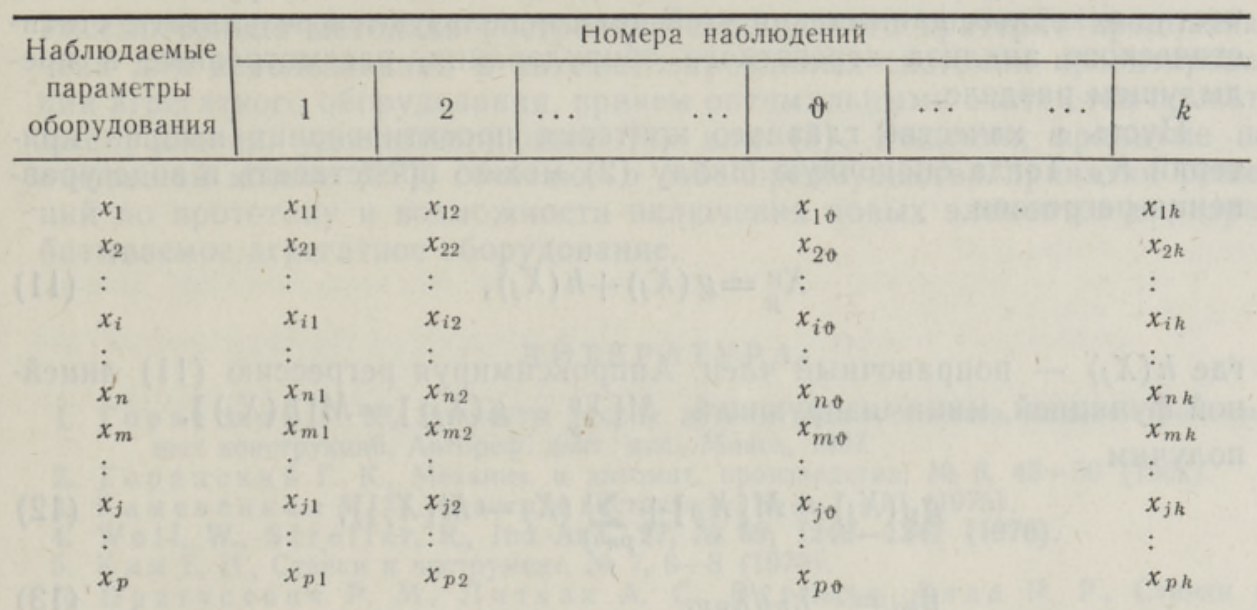

$$
\begin{aligned}
& r_{i(i+1)}=\left(k \sigma_{i} \sigma_{i+1}\right)^{-1} \sum_{\vartheta=1}^{k}\left(x_{i}-M\left[x_{i}\right]\right)\left(x_{i+1}-M\left[x_{i+1}\right]\right), \\
& r_{j(j+1)}=\left(k \sigma_{j} \sigma_{j+1}\right)^{-1} \sum_{\vartheta=1}^{k}\left(x_{j}-M\left[x_{j}\right]\right)\left(x_{j+1}-M\left[x_{j+1}\right]\right), \\
& r_{i j}=\left(k \sigma_{i} \sigma_{j}\right)^{-1} \sum_{0=1}^{k}\left(x_{i}-M\left[x_{i}\right]\right)\left(x_{j}-M\left[x_{j}\right]\right) .
\end{aligned}
$$

По результатам вычисления строится корреляционная матрица, симметричная относительно главной ддиагонали $\vartheta_{i j}$.

Значения коэффициентов парной корреляции оценочных параметров $\vartheta_{i j}$ расположены на отрезке $-1 \leqslant \vartheta_{i j} \leqslant 1$, а предельные случаи $\theta_{i j}=0$ и $\left|\vartheta_{i j}\right|=1$ соответствуют статистической независимости и линейной зависимости, причем если $\vartheta_{i j}>0$, то с увеличением $i$ величина $j$ в среднем возрастает, а в противном случае - в среднем убывает. Это свойство коэффициентов корреляции определяет направление устремления частных критериев проектирования в обобщенном критерии. Отметим, что использование коэффициентов корреляции как меры взаимосвязи между характеристиками оборудования возможно только в случае нормального распределения. При других видах распределений они становятся ненадежными. Для проверки распределения в плане его соответствия нормальному закону строятся гистограммы или используется критерий Колмогорова-Смирнова. Однако, как показано в [ $\left.{ }^{11}\right]$, большинство параметров агрегатного оборудования подчиняются нормальному закону распределения.

\section{4. Статистическое моделирование оценочной функции}

Задание коэффициентов важности в обобщенном критерии проектирования - весьма ответственная и трудоемкая операция. Традиционно используемые экспертные методы и методы «голосования» $[9,19,20]$ сложны и субъективны по своей сути, что приводит к недостаточно обоснованному заданию коэффициентов важности. Упростить анализ и 
повысить адекватность оценочной функции обобщенного критерия можно путем статистического моделирования оценочной функции. В качестве исходных данных для этой цели используются результаты статистического анализа агрегатного оборудования, рассмотренные в предыдущем разделе.

Пусть в качестве главного критерия проектирования выбран критерий $K_{p}$. Тогда оценочную шкалу (2) можно представить в виде уравнения регрессии

$$
X_{p}^{\mathrm{\kappa}}=g\left(X_{j}\right)+h\left(X_{j}\right)
$$

где $h\left(X_{j}\right)$ - поправочный член. Аппроксимируя регрессию (11) линейной функцией, минимизирующей $M\left[X_{p}^{\mathrm{k}}-g\left(X_{j}\right)\right]=M\left[h\left(X_{j}\right)\right]$, получим

$$
\begin{aligned}
& g_{p}\left(X_{j}\right)=M\left[X_{p}\right]+\sum_{p \neq j}\left(X_{j}-M\left[X_{j}\right]\right), \\
& \beta_{p j}=-\lambda_{p j} / \lambda_{p p},
\end{aligned}
$$

где $\lambda_{p j}$ - матрица, обратная матрице центральных моментов. Уравнение (12) является статистической моделью оценочной шкалы (2), а (13) определяет статистический коэффициент важности обобщенного критерия. Тогда (11) можно представить в виде

$$
X_{p}^{\mathrm{\kappa}}=M\left[X_{p}\right]+\sum_{p \neq j}\left(X_{j}-M\left[X_{j}\right]\right) .
$$

Статистическая оценочная шкала прогноза главного критерия строится аналогично:

$$
\begin{aligned}
& X_{p}^{\mathrm{I}}=M\left[X_{p}\right]+\sum_{p \neq i}\left(X_{i}-M\left[X_{i}\right]\right), \\
& \beta_{p i}=-\lambda_{p i} / \lambda_{p p} .
\end{aligned}
$$

Подставляя выражения (14) и (15) в соотношение (3), получим статистическую модель оценочной функции обобщенного критерия, учитывающую прогнозируемые и конечные конструктивные параметры

$$
\begin{gathered}
\eta_{x}=M\left[X_{p}\right]+\sum_{p \neq i} \beta_{p i}\left(X_{i}-M\left[X_{i}\right]\right) / M\left[X_{p}\right]+\sum_{p \neq j} \beta_{p j}\left(X_{j}-M\left[X_{j}\right]\right), \\
\beta_{p i} \neq \beta_{p j} .
\end{gathered}
$$

Для конкретного технического задания на проектирование агрегатного оборудования числитель и $k=j-n$ оценочных параметров уравнения (17) можно представить в виде

$$
\eta_{x}=C\left[A+\sum_{p \neq k} \beta_{p k}\left(X_{k}-M\left[X_{k}\right]\right)\right]
$$

где $A$ и $C$ - постоянные для конкретной исходной информации на проектирование.

Уравнение (19) описывает статистическую оценочную функцию для конечных конструктивных параметров проектируемого агрегатного оборудования с коэффициентами важности частных критериев. 


\section{5. Заключение}

Рассмотренная методика построения обобщенного критерия предназначена для использования в автоматизированных системах проектирования агрегатного оборудования, причем оптимальными считаются проектные решения, удовлетворяющие (4) или (5). Решения, принятые по оценочной шкале (17), сочетают в себе преимущества принятия решений по прототипу и возможности включения новых элементов в разрабатываемое агрегатное оборудование.

\section{Л И Т Е Р А Т У Р А}

1. Гор ански й Г. К., Элементы теории автоматизации синтеза машиностроительных конструкций, Автореф. докт. дис., Минск, 1967.

2. Го р ан ский Г. К., Механиз. и автомат. производства, № 6, 45-50 (1962).

3. К а м ен ец к а я М. П., Станки и инструмент, № 9, 7-10 (1975).

4. Wolf, W., Strelle r, R., Ind.-Anz., 27, № 69, 1246-1247 (1976).

5. К и м Е. Н., Станки и инструмент, № 7, 6-8 (1979).

6. Пратусевич Р. М., Литвак А. С., Бургете Аяла Р. Р., Станки и инструмент, № 7, 3-6 (1979).

7. Современное состояние исследования операций (под ред. Н. Н. Моисеева), М., «Наука», 1979.

8. Г ермейер Ю. Б., Введение в теорию исследования операций, М., «Наука», 1971.

9. Ге ермей ер Ю. Б., Изв. АН СССР, Техн. кибернетика, № 4, 3-13 (1976).

10. О з е рн о й В. М., Автоматика и телемеханика, № 12, 106-121 (1971).

11. Н а йдин Ю. В., Проектирование многошпиндельных коробок агрегатных станков с помощью ЭВМ, Автореф. канд. дис., М., 1969.

12. Ц а р е в А. М., Изв. ВУЗов, Машиностроение, № 4, 178-182 (1978).

13. Подин ов ский В. В., Автоматика и телемеханика, № $10,130-141$ (1978).

14. Автоматизация проектно-конструкторских работ и технологической подготовки производства в машиностроении, т. 1 и 2 (под ред. О. И. Семенкова), Минск, «Вышэйш. школа», 1976.

15. Е мельяно в С. В. и др., В кн.: Техническая кибернетика. Итоги науки и техники, М., изд. ВИНИТИ 5,1973 , с. $386-448$.

16. Ор лов П. И., Основы конструирования, М., «Машиностроение», 1977.

17. П уш В. Э., Конструирование металлорежущих станков, М., «Машиностроение», 1977.

18. Мит ро польский А. К., Техника статистических вычислений, М., «Статистика», 1962.

19. Л а р иче в О. И., Автоматика и телемеханика, № 12, 130-142 (1971).

20. Глотов В. А., П а вельев В. В., Автоматика и телемеханика, № 12, 95-107 (1976).

Таллинский политехнический институт
Поступила в редакцию 1/VII 1980

\section{SENDRIK}

\section{OHEST OLDISTATUD EFEKTIIVSUSKRITEERIUMI SUNTEESI MEETODIST AGREGAATSEADMETE AUTOMATISEERITUD PROJEKTEERIMISE SUSTEEMIDES}

Artikkel käsitleb küsimusi, mis on seotud agregaatseadmete projekteerimise üldistatud kriteeriumi formeerimisega juhul, kui projekteerimise üldine sihifunktsioon muutub iga konkreetse projekti puhul. On esitatud üldistatud kriteeriumi statistiliste kaalutegurite määramise metoodika ja koostatud võrrandid prognoosivale ja aposterioorsele hinnangufunktsioonile. 
M. SENDRIK

\section{METHOD OF SYNTHESIS OF COLLECTED EFFICIENCY CRITERION IN COMPUTER-AIDED AGGREGATE EQUIPMENT DESIGN}

The paper describes a method for formulating the collected efficiency criterion in computer-aided aggregate equipment design in case of design goal function changing from task to task. The collected efficiency criterion is derived from the design criterion set $K=\left\{K_{i}\right\}^{p}=1$ as one single function $\Phi\left(\alpha_{i}, K_{i}\right)$, where $\forall K_{i} \in K$ is selected depending on the design goal.

A method of determining weight coefficients $\alpha_{i}$ of the above-mentioned criterion by statistic analysis is given.

The equations of prognosing as well as a posteriori estimation functions are presented.

The proposed collected efficiency criterion takes into account the complexity of the particular designing task as well as the corresponding optimal conditions. 